\title{
Polimer-Polimer Karışabilirliğinin Guguk Kuşu Arama Algoritmasına Dayalı Ağırlıklı Bulanık Sınıflandırma Sistemiyle Tahmini
}

\author{
Gözde Güldiken ${ }^{1 *}$, Mehmet Levent Koç², Dilek İmren Koç \\ ${ }^{1}$ Sivas Cumhuriyet Üniversitesi, Mühendislik Fakültesi, Kimya Mühendisliği Bölümü, Sivas, Türkiye, (ORCID: 0000-0002-4510-5303), guldikengozde@gmail.com \\ ${ }^{2}$ Sivas Cumhuriyet Üniversitesi, Mühendislik Fakültesi, İnşaat Mühendisliği Bölümü, Sivas, Türkiye (ORCID: 0000-0003-4993-6152), \\ mkoc@cumhuriyet.edu.tr \\ ${ }^{3}$ Sivas Cumhuriyet Üniversitesi, Mühendislik Fakültesi, Kimya Mühendisliği Bölümü, Sivas, Türkiye (ORCID: 0000-0003-3657-3794), dimren@,cumhuriyet.edu.tr
}

(1st International Conference on Applied Engineering and Natural Sciences ICAENS 2021, November 1-3, 2021)

(DOI: 10.31590/ejosat.1008608)

ATIF/REFERENCE: Güldiken G., Koç M.L., İmren Koç D. (2021). Polimer-Polimer Karışabilirliğinin Guguk Kuşu Arama Algoritmasına Dayalı Ağırlıklı Bulanık Sınıflandırma Sistemiyle Tahmini. Avrupa Bilim ve Teknoloji Dergisi, (28), $519-525$.

$\ddot{\mathbf{O z}}$

Bu çalışma, "ağırlıklı bulanık sınıflandırma sistemi" ile guguk kuşu aramasının birleşiminden oluşan yeni bir hibrit bulanık sınıflandırma sistemini çözelti fazındaki sodyum aljinat/poli(etilen glikol) karışımların karışabilirlik tahmini için önermekte ve söz konusu bulanık sistemin tahmin etme yeteneğini incelemektedir. Test sonuçları, önerilen bulanık sistemin, iki farklı bulanık sisteme (Mamdani-Tip ve Sugeno-Tip) ve etkileşim parametrelerini $(\alpha, \Delta \eta$ and $\beta$ ) kullanan geleneksel viskometrik analize kıyasla, daha yüksek doğru sınıflandırma oranı elde ettiğini göstermiştir.

Anahtar Kelimeler: Bulanık Sistem, Guguk Kuşu Araması, Polimer, Karışabilirlik, Viskozimetri

\section{Prediction of Polymer-Polymer Miscibility by Cuckoo Search Algorithm Based Fuzzy Classification System}

\begin{abstract}
This study proposes a new hybrid fuzzy classification system which combines "weighting fuzzy classification system" with cuckoo search algorithm in order to predict the miscibility of sodium alginate/poly(ethylene glycol) blends in solution phase and examines its predictive capability. The test results show that the proposed fuzzy system gets a more accurate classification rate than the two types of fuzzy systems (Mamdani-Type and Sugeno-Type) as well as traditional viscometric analysis using interaction parameters $(\alpha, \Delta \eta$ and $\beta)$.
\end{abstract}

Keywords: Fuzzy System, Cuckoo Search, Polymer, Miscibility, Viscometry

* Gözde Güldiken: guldikengozde@gmail.com 
$R_{j}:$ Ĕger $x_{1} A_{j 1}$ ve ... ve $x_{n} A_{j n}$ ise sinuf $C F_{j}$ ile $C_{j}$ dir
$j=1,2, \ldots, N$

\section{Giriş}

Son yıllarda yapılan bazı çalışmaların ortaya koyduğu sonuçlar, polimer-polimer karışabilirliğini tahmin etmek amacıyla "bulanıklık" altında gerçekleştirilecek viskozimetrik analizleri yeni çalışmalarla desteklenmesi gereken güncel araştırma konuları haline getirmiştir: Koç ve Koç (2016) klasik viskozimetrik analizi "bulanıklaştırma" aracı olarak temelde bulanık regresyonu kullanmışlar; Koç et al., (2019) ise bulanık (Mamdani, 1977) ve nöro-bulanık (Takagi, Sugeno, 1985) sistemleri önermişler ve bulanık regresyona/nöro-bulanık sisteme kıyasla bulanıklaştırmanın ve bulanıklık altında işlem yapmanın daha kolay ve hesaplama yönünden daha az maliyetli yolları olan bulanık sistemlerin, bu avantajlarına karşın, tahmin (sınıflandırma) yeteneklerinin geliştirilmelerine duyulan ihtiyacı vurgulamışlardır. Bulanık sistemlerin sınıflandırma yeteneklerinin iyileştirilmesine dönük temel yaklaşımlardan biri bulanık kural tabanının veri tabanı üzerinden, uzman bilgisine dayanmaksızın, kendiliğinden çıkarımı olup bu kapsamda önerilen yöntemlere örnek olarak "ağırlıklı bulanık sinıflandırma sistemi" (Ozkaya ve Seyfi, 2016; Nakashima et al., 2007) verilebilir. Burada, söz konusu yöntem, esas olarak, benzerlerinden sisteme ait girdilerin ağırlık faktörleriyle ayrıştırılmasıyla farklılık göstermekte ve amaç fonksiyonunun en küçüklemesi ile bulanık kural tabanının çıkarımına dayanmaktadır. En küçükleme işlemi için uygun optimizasyon tekniklerinden biri guguk kuşlarının "kuluçka asalaklığı" davranışını taklit eden guguk kuşu arama algoritması(Yang and Deb, 2013; Yang and Deb, 2014) olup görece yeni sayılabilen bu meta-sezgisel algoritmanın yaygın kullanılan genetik algoritma, parçacık sürü optimizasyonu, yapay arı koloni algoritması gibi tekniklere kıyasla daha iyi çözüm performansına sahip olduğu (Yang and Deb, 2013; Nawi et al., 2013; Kumar and Rawat, 2015; Samir et al., 2018; Ebenezer et al., 2019) ve daha önce klasik bulanık sinıflandırma sistemlerinin optimal tasarımında (Hodashinsky, 2015; Ding et al., 2015) sınırlı olarak kullanıldığı söylenebilir. Bu çalışmada, ağırlıklı bulanık sinıflandırma sistemi, sodyum aljinat/poli(etilen glikol) (SA/PEG) karışımlar örneğinde (Koç et al.,2019), guguk kuşu arama algoritmasına dayalı olarak polimer-polimer karışabilirlik tahminine literatürde ilk kez uygulanmış ve bu yolla i)bulanık sistemlerin karışabilirlik tahmin/sınıflandırma yeteneklerinin iyileştirilme olanaklarının araştırılması, ii)farklı alanlardaki bulanık sınıflandırma problemlerinin çözümüne de yardımcı olabilecek yeni bir hibrit bulanık sınıflandırma yaklaşımı ortaya konması amaçlanmıştır.

\section{Yöntem: Ă̆ırlıklı Bulanık Sınıflandırma Sistemi}

Ağırlıklı bulanık sinıflandırma sistemi (ABSS), "ağırlık faktörleri" ile "ağırlıklandırılmış"bir eğitme veri kümesinden bulanık kural tabanının kendiliğinden çıkarımına dayanan, sınıflandırma amaçlı, bulanık kural tabanlı bir sistem olarak tanımlanabilir ve bulanık kural tabanındaki bir kural $\left(R_{j}\right)$, nboyutlu bir sınıflandırma problemi için, aşağıdaki şekilde verilebilir:
Burada, $x_{i}$ sistemin girdi değişkeni $(i=1,2, \ldots, n), A_{j i}$ girdi değişkenine ait bulanık alt küme $(i=1,2, \ldots, n ; j=1,2, \ldots, N)$ ve $C F_{j}$ (j. kural için kesinlik derecesi) ile $C_{j}$ (j. kural için göz önüne alınan probleme ait sinıflardan herhangi biri) sistemin çıktıları olup aşağıda verilen adımlarla belirlenebilir:

1. Adım: eğitme veri kümesine ait her bir girdi vektörü $\left(X_{p}=\right.$ $\left.\left(x_{p 1}, \ldots, x_{p n}\right)\right)$ için bulanık alt kümelerin $\left(A_{j i}^{k}\right)$ üyelik fonksiyonları $\mu_{j i}(\cdot)$ yardımıyla kurala ait $\mu_{j}\left(X_{p}\right)$ değerini hesapla $(k=1, \ldots, z)$ :

$\mu_{j}\left(X_{p}\right)=\mu_{j 1}\left(x_{p 1}\right) \times \cdots \times \mu_{j n}\left(x_{p n}\right)$, $p=1,2, \ldots, m$

Bu çalışmada, girdi değişkenlerine ait toplam bulanık alt küme sayısı ( $z$ ) dört olarak seçilmiş olup $k=1,2,3$ ve 4 olarak etiketlenen bulanık alt kümeler için üyelik fonksiyonları sırasıyla aşağıdaki şekillerde tanımlanmıştır:

$\mu_{j i}\left(x_{p i}\right)=\left\{\begin{array}{cc}1-e^{-\frac{1}{2}\left(\frac{x_{p i}-c_{i}}{\sigma_{i}}\right)^{2}} x_{p i} \leq c_{i} \\ 0 & x_{p i}>c_{i}\end{array}\right.$

$\mu_{j i}\left(x_{p i}\right)=e^{-\frac{1}{2}\left(\frac{x_{p i}-c_{i}}{\sigma_{i}}\right)^{2}}$

$\mu_{j i}\left(x_{p i}\right)= \begin{cases}1-e^{-\frac{1}{2}\left(\frac{x_{p i}-c_{i}}{\sigma_{i}}\right)^{2}} & x_{p i} \geq c_{i} \\ 0 & x_{p i}<c_{i}\end{cases}$

$\mu_{j i}\left(x_{p i}\right)=1$

2. Adım: probleme ait her bir sınıf $(h)$ için $\beta_{h}\left(R_{j}\right)$ değerini eğitme veri kümesi ağırlık faktörleri $\left(w_{p}\right)$ yardımıyla hesapla:

$$
\begin{aligned}
& \beta_{h}\left(R_{j}\right)=\sum_{X_{p} \epsilon h} \mu_{j 1}\left(x_{p 1}\right) \ldots \mu_{j n}\left(x_{p n}\right) w_{p}, h \\
& =1,2, \ldots, C
\end{aligned}
$$

3. Adım: $\beta_{\dot{h}}\left(R_{j}\right)=\max \left(\beta_{1}\left(R_{j}\right), \ldots, \beta_{C}\left(R_{j}\right)\right)$ için sınıfı $\left(C_{j}\right) \dot{h}$ olarak belirle ve $C F_{j}$ değerini hesapla:

$C F_{j}=\frac{\beta_{\dot{h}}\left(R_{j}\right)-\bar{\beta}}{\sum \beta_{h}\left(R_{j}\right)}$ 
$\bar{\beta}=\frac{\sum_{h \neq \dot{h}} \beta_{h}\left(R_{j}\right)}{C-1}$

$N$ adet kuraldan oluşan bulanık kural tabanının göz önüne alınması halinde ise bir girdi vektörü $X=\left(x_{1}, x_{2}, \ldots, x_{n}\right)$ için bulanık sistemin çıktısı (probleme ait sınıflardan herhangi biri) aşağıda verilen adımlarla belirlenebilir:

1. Adım: probleme ait her bir $\operatorname{sinıf}(h=1,2, \ldots, C)$ için $\delta_{h}(X)$ değerini hesapla:

$\delta_{h}(X)=\max \left\{\mu_{j}(X) C F_{j} \mid C_{j}=h\right\}$

2. Adım: $\delta_{h^{*}}(X)=\max \left(\delta_{1}(X), \ldots, \delta_{C}(X)\right)$ için sistemin çıktısını $h^{*}$ olarak belirle ve birden fazla sınıf için $\delta_{h^{*}}$ değeri eşit ise göz önüne alınan girdi vektörünü "sınıflandırılamaz" olarak etiketle.

Bulanık kural tabanının bir eğitme veri kümesinden (ağırlıklandırılmış) kendiliğinden çıkarımı, uygun bir "eğitme algoritması" yardımıyla aşağıda verilen amaç fonksiyonunun $(F)$ minimizasyonu ile sağlanabilir:

$$
F(S)=\sum_{p=1}^{m} w_{p} Z_{p}(S)
$$

Burada, $Z_{p}(S)$ bulanık sistemin (S) çıktısı (sınıf) doğru ise $Z_{p}(S)=0$ ve aksi durumda $Z_{p}(S)=1$ değerini alan ikili bir değişken iken $w_{p}$ eğitme veri kümesi için ağırlık faktörü olarak tanımlanabilir. Bu çalışmada, eğitme veri kümesi "sınıf tabanlı ağırlıklandırma" yöntemiyle (Nakashima et al., 2005) ağırlıklandırılmış $\left(w_{p}=\left(w_{p}^{1}, \ldots, w_{p}^{h}, \ldots, w_{p}^{C}\right)\right)$ olup bulanık siniflandırma sisteminin parametre vektörü, $\boldsymbol{S}=$ $\left(w_{p}^{1}, \ldots, w_{p}^{C}, c_{1}, \ldots, c_{n}, A_{11}^{k}, \ldots, A_{1 n}^{k}, A_{21}^{k}, \ldots, A_{N n}^{k}\right)$, üzerinden tanımlanan amaç fonksiyonu, $F(S)=\sum_{p=1}^{m} w_{p} Z_{p}(S)$, guguk kuşu arama algoritması ile en küçüklenmiştir. Guguk kuşu arama algoritması, üç temel kurala dayalı olarak, guguk kuşlarının agresif üreme stratejilerini taklit eden bir optimizasyon tekniği olarak tanımlanabilir (Yang and Deb, 2013; Yang and Deb, 2014): 1) her bir guguk kuşu bir seferde bir yumurtayı rastgele seçilmiş bir yuvaya bırakır, 2) yüksek kaliteli yumurtalara veya çözümlere sahip en iyi yuvalar sonraki jenerasyonlara aktarılır, 3) uygun konak yuva sayısı sabittir ve konak kuşun, guguk kuşu tarafindan bırakılan bir yumurtayı bir $P_{a} \in[0,1]$ olasıllı̆ 1 ile fark etmesiyle yumurta konak kuş tarafindan yuvadan atılır ya da yuva konak kuş tarafından terk edilir. Guguk kuşu arama algoritmasının eğitme aşaması uygulaması için kaba-kod aşağıda verilmiştir:

$K$ adet konak yuva $\boldsymbol{S}_{i}$ için başlangıç popülasyonunu rastgele üret ve uygunluk/amaç fonksiyonu $F_{i}$ değerlerini hesapla $(i=$ $1, \ldots, K)$
While(durdurma kriteri)

Lévy uçuşu ile rastgele bir guguk kuşu al (varsayalım $j$ ) ve buna ait uygunluk değerini $F_{j}$ hesapla.

$K$ Yuva arasından rastgele bir yuva seç (varsayalım $i$ )

$$
\begin{aligned}
& \text { if } F_{j}<F_{i} \text { then } \\
& \boldsymbol{S}_{i} \leftarrow \boldsymbol{S}_{j} \\
& F_{i} \leftarrow F_{j}
\end{aligned}
$$

\section{end if}

Fraksiyon değerine $\left(P_{a}\right)$ göre kötü yuvaları terk et ve Lévy uçuşu ile yenilerini inşa et.

Yeni yuvaların uygunluklarını amaç fonksiyonuna göre değerlendir.

En iyi yuvaları/çözümleri sakla.

Çözümleri sırala ve mevcut en iyi çözümü bul.

\section{end while}

Burada, Lévy uçuşu, Lévy dağılımı ile karakterize edilen bir tür "rassal yürüyüş" olarak tanımlanabilir (Yang and Deb, 2013) ve yeni çözümlerin aranması sırasında uçuşun ölçeği, adım büyüklügü faktörü $(\alpha>0)$ ile kontrol edilir. Bu çalışmada, $P_{a}$ ve $\alpha$ değerleri sırasıyla 0,2 ve 0,01 olarak seçilmiş olup durdurma kriteri olarak maksimum jenerasyon sayıs 1000 olarak alınmıştır.

\section{Bulgular ve Tartışma}

SA/PEG sulu çözelti karışımlarının karışabilirlik tahminine yönelik olarak sunulan ABSS tipi bulanık sistemin eğitme/test aşamasında farklı derişim aralıkları $(0,1-0,5 \mathrm{~g} / \mathrm{dL})$, karışım oranlar1 $(10 / 90,30 / 70,50 / 50,70 / 30$ ve $90 / 10$ SA/PEG-4000 ve SA/PEG-8000) ve sicaklıklar $\left(30^{\circ} \mathrm{C}\right.$ ve $\left.50{ }^{\circ} \mathrm{C}\right)$ için elde edilen $\alpha, \Delta \eta$ ve $\beta$ klasik etkileşim parametreleri (Tablo 1) ile birlikte refraktif indeks (Şekil 1 ve 2) değerlerinden (Koç ve ark., 2019) yararlanılmıştır. Bulanık sınıflandırma sisteminin girdileri $\alpha$ ve $\beta$ etkileşim parametreleri olup bu girdi değişkenleri sırasıyla $\boldsymbol{\alpha}_{1}(k=1), \boldsymbol{\alpha}_{2}(k=2), \boldsymbol{\alpha}_{3}(k=3), \boldsymbol{\alpha}_{4}(k=4)$ ve $\boldsymbol{\beta}_{1}(k=1), \boldsymbol{\beta}_{2}(k=2)$, $\boldsymbol{\beta}_{3}(k=3), \quad \boldsymbol{\beta}_{4}(k=4) \quad$ bulanık alt kümeleriyle/üyelik fonksiyonlarıyla (Eşitlik 3-6) temsil edilirken; KRZ (karışmaz) ve KR (karışabilir) sınıfları sistemin çıktısını oluşturmuş̧ur.

Şekil 1.Refraktif indeks değerlerinin SA/PEG-4000 karışımları için SA içeriği ile a) $30^{\circ} \mathrm{C}$ ve b) $50^{\circ} \mathrm{C}$ 'de değiş̧imi (Koç ve ark. 2019)

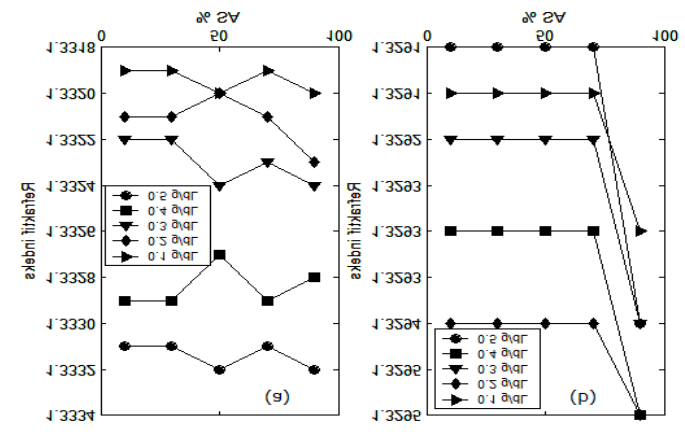


Şekil 2. Refraktif indeks değerlerinin SA/PEG-8000 karışımları için $\mathrm{SA}$ içeriği ile a) $30^{\circ} \mathrm{C}$ ve b) $50{ }^{\circ} \mathrm{C}$ 'de değişimi (Koç ve ark. 2019)

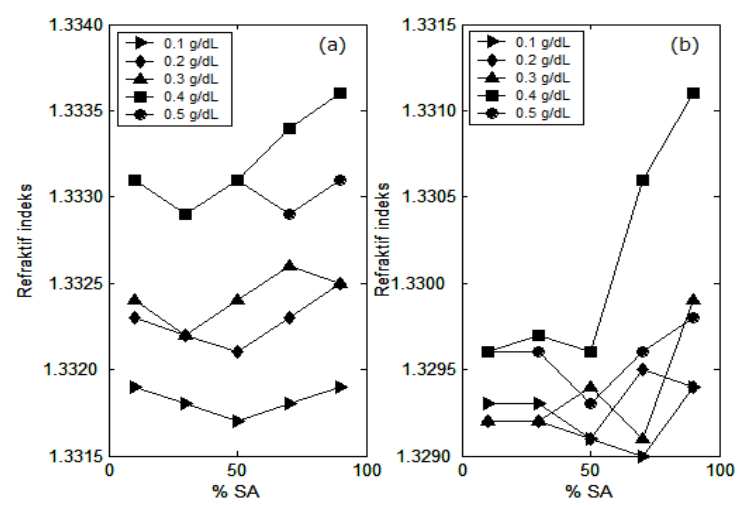

Tablo 1'de verilen veri kümesinin \% 60'1 eğitme kalan kısmı ise test aşamasında kullanılmış ve eğitme aşaması (Şekil 3) sonucunda elde edilen parametre vektörüne karşıllk gelen üyelik fonksiyonları Şekil 4 ve 5'de; bulanık kural tabanı ise aşağıda verilmiştir.

$$
\begin{aligned}
& R_{1}: \text { Ĕger } \alpha \alpha_{1} \text { ve } \beta \boldsymbol{\beta}_{2} \text { ise sinıf } C F_{1}=0.999 \text { ile } K R Z \text { dir } \\
& R_{2}: \text { Ĕger } \alpha \alpha_{2} \text { ve } \beta \boldsymbol{\beta}_{3} \text { ise sinıf } C F_{2}=0.269 \text { ile } K R \text { dir } \\
& R_{3}: \text { Ĕger } \alpha \alpha_{3} \text { ve } \beta \boldsymbol{\beta}_{2} \text { ise sinıf } C F_{3}=0.136 \text { ile } K R Z \text { dir } \\
& R_{4}: \text { Ĕger } \alpha \alpha_{2} \text { ve } \beta \boldsymbol{\beta}_{1} \text { ise sinıf } C F_{4}=1.000 \text { ile } K R Z \text { dir }
\end{aligned}
$$

Şekil 3. Eğitme aşaması için amaç fonksiyonunun jenerasyon

\begin{tabular}{|c|c|c|c|c|c|c|c|c|c|c|}
\hline & SA İçeriği & Sira & $\mathrm{C}$ & & $30^{\circ} \mathrm{C}$ & & Sira & & & \\
\hline & & No & $(\mathrm{g} / \mathrm{dL})$ & $\alpha$ & $\Delta \eta$ & $\beta$ & No & $\alpha$ & $\Delta \eta$ & $\beta$ \\
\hline & & $1 *$ & 0.1 & 1.114 & 5.023 & 0.4845 & $51^{*}$ & 1.753 & 3.802 & 0.691 \\
\hline & & 2 & 0.2 & 0.455 & 4.765 & 0.2207 & 52 & 1.005 & 3.526 & 0.470 \\
\hline & $10 / 90$ & 3 & 0.3 & 0.253 & 4.501 & 0.1281 & 53 & 0.426 & 3.347 & 0.212 \\
\hline & & 4 & 0.4 & 0.201 & 4.183 & 0.0994 & 54 & 0.331 & 2.988 & 0.167 \\
\hline & & $5 *$ & 0.5 & 0.101 & 3.582 & 0.0471 & 55 & 0.163 & 2.481 & 0.082 \\
\hline & & 6 & 0.1 & 1.606 & 7.120 & 0.7359 & 56 & 1.380 & 7.050 & 0.675 \\
\hline & & $7 *$ & 0.2 & 0.701 & 6.440 & 0.2783 & 57* & 0.588 & 6.324 & 0.248 \\
\hline & $30 / 70$ & 8 & 0.3 & 0.457 & 5.583 & 0.1445 & 58 & 0.389 & 5.774 & 0.142 \\
\hline & & 9* & 0.4 & 0.213 & 4.648 & 0.0549 & $59 *$ & 0.204 & 4.734 & 0.058 \\
\hline & & 10 & 0.5 & 0.105 & 3.024 & 0.0217 & $60 *$ & 0.087 & 3.622 & 0.022 \\
\hline & & 11 & 0.1 & -0.014 & 14.113 & -0.0018 & 61 & 0.045 & 11.493 & 0.032 \\
\hline SA/PEG4000 & & $12 *$ & 0.2 & -0.005 & 13.011 & -0.0030 & $62 *$ & 0.041 & 10.319 & 0.010 \\
\hline & $50 / 50$ & $13 *$ & 0.3 & 0.000 & 11.561 & -0.0025 & 63 & 0.023 & 9.397 & 0.002 \\
\hline & & 14 & 0.4 & -0.002 & 10.010 & -0.0023 & 64 & 0.014 & 7.676 & -0.001 \\
\hline & & $15 *$ & 0.5 & -0.001 & 7.363 & -0.0014 & 65 & 0.001 & 5.959 & -0.002 \\
\hline & & 16 & 0.1 & -0.095 & 18.392 & -0.0139 & $66^{*}$ & 0.079 & 14.318 & 0.028 \\
\hline & & $17 *$ & 0.2 & -0.045 & 16.867 & -0.0066 & 67 & 0.077 & 12.694 & 0.010 \\
\hline & $70 / 30$ & 18 & 0.3 & -0.022 & 14.824 & -0.0032 & 68 & 0.044 & 11.400 & 0.003 \\
\hline & & $19 *$ & 0.4 & -0.013 & 12.657 & -0.0019 & 69 & 0.017 & 8.999 & 0.000 \\
\hline & & 20 & 0.5 & -0.006 & 8.987 & -0.0010 & 70 & 0.005 & 6.677 & -0.001 \\
\hline & & $21 *$ & 0.1 & -0.051 & 21.223 & -0.0019 & 71 & -0.183 & 18.429 & -0.006 \\
\hline & & 22 & 0.2 & -0.022 & 19.275 & -0.0011 & $72 *$ & -0.069 & 16.356 & -0.003 \\
\hline & $90 / 10$ & 23 & 0.3 & -0.009 & 16.639 & -0.0006 & 73 & -0.039 & 14.691 & -0.002 \\
\hline & & 24 & 0.4 & -0.006 & 13.856 & -0.0004 & $74 *$ & -0.019 & 11.610 & -0.001 \\
\hline & & $25^{*}$ & 0.5 & -0.003 & 9.163 & -0.0002 & 75 & -0.012 & 8.682 & -0.001 \\
\hline & & 26 & 0.1 & 1.292 & 5.855 & 0.5686 & $76^{*}$ & 0.909 & 3.984 & 0.3818 \\
\hline & & 27 & 0.2 & 0.799 & 5.557 & 0.3920 & $77 *$ & 0.576 & 3.812 & 0.3162 \\
\hline & $10 / 90$ & $28^{*}$ & 0.3 & 0.384 & 5.233 & 0.2020 & $78^{*}$ & 0.281 & 3.604 & 0.1748 \\
\hline & & $29 *$ & 0.4 & 0.129 & 4.832 & 0.0720 & $79 *$ & 0.079 & 3.193 & 0.0623 \\
\hline & & 30 & 0.5 & 0.119 & 4.301 & 0.0610 & $80 *$ & 0.076 & 2.640 & 0.0521 \\
\hline & & $31 *$ & 0.1 & -1.187 & 12.582 & -0.5524 & 81 & 0.421 & 6.804 & 0.1985 \\
\hline & & $32 *$ & 0.2 & -0.574 & 11.870 & -0.2428 & 82 & 0.349 & 6.160 & 0.1263 \\
\hline & $30 / 70$ & 33 & 0.3 & -0.408 & 10.967 & -0.1483 & 83 & 0.094 & 5.586 & 0.0186 \\
\hline & & $34 *$ & 0.4 & -0.223 & 9.968 & -0.0738 & 84 & 0.060 & 4.506 & 0.0063 \\
\hline
\end{tabular}
sayısına bă̆lı değişimi

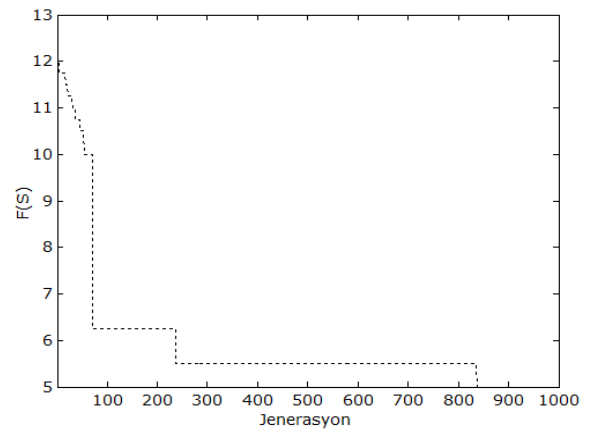

Tablo 1. SA/PEG karışımları için etkileşim parametreleri (Koç ve ark. 2019) 
European Journal of Science and Technology

\begin{tabular}{|c|c|c|c|c|c|c|c|c|c|c|}
\hline \multirow{16}{*}{ SA/PEG8000 } & & 35 & 0.5 & -0.143 & 8.399 & -0.0389 & 85 & 0.042 & 3.359 & 0.0048 \\
\hline & \multirow{5}{*}{$50 / 50$} & 36 & 0.1 & -0.040 & 14.717 & -0.0210 & $86^{*}$ & -0.163 & 12.340 & -0.0732 \\
\hline & & $37 *$ & 0.2 & -0.030 & 13.592 & -0.0151 & 87 & -0.071 & 11.223 & -0.0379 \\
\hline & & $38^{*}$ & 0.3 & -0.014 & 12.109 & -0.0083 & 88 & -0.048 & 10.285 & -0.0255 \\
\hline & & 39 & 0.4 & -0.008 & 10.513 & -0.0051 & 89 & -0.023 & 8.536 & -0.0135 \\
\hline & & $40^{*}$ & 0.5 & -0.004 & 7.905 & -0.0029 & 90 & -0.015 & 6.793 & -0.0083 \\
\hline & \multirow{5}{*}{$70 / 30$} & 41 & 0.1 & -0.050 & 18.365 & -0.0147 & $91^{*}$ & 0.880 & 13.493 & 0.1632 \\
\hline & & 42 & 0.2 & -0.024 & 16.826 & -0.0081 & 92 & 0.282 & 11.904 & 0.0226 \\
\hline & & 43 & 0.3 & -0.013 & 14.764 & -0.0045 & $93 *$ & 0.114 & 10.600 & 0.0023 \\
\hline & & $44^{*}$ & 0.4 & -0.008 & 12.569 & -0.0027 & 94* & 0.074 & 8.182 & 0.0004 \\
\hline & & 45 & 0.5 & -0.003 & 8.923 & -0.0014 & 95 & 0.048 & 5.845 & 0.0003 \\
\hline & \multirow{5}{*}{$90 / 10$} & 46 & 0.1 & -0.081 & 21.966 & -0.0063 & 96 & 0.839 & 15.031 & 0.0469 \\
\hline & & $47^{*}$ & 0.2 & -0.038 & 20.014 & -0.0030 & 97 & 0.319 & 12.969 & 0.0074 \\
\hline & & 48 & 0.3 & -0.018 & 17.372 & -0.0015 & 98 & 0.152 & 11.301 & 0.0015 \\
\hline & & 49 & 0.4 & -0.010 & 14.580 & -0.0008 & 99* & 0.083 & 8.214 & 0.0002 \\
\hline & & 50 & 0.5 & -0.005 & 9.895 & -0.0004 & $100 *$ & 0.041 & 5.282 & -0.0001 \\
\hline
\end{tabular}

Şekil 4. $\alpha$ için bulanık alt kümeler

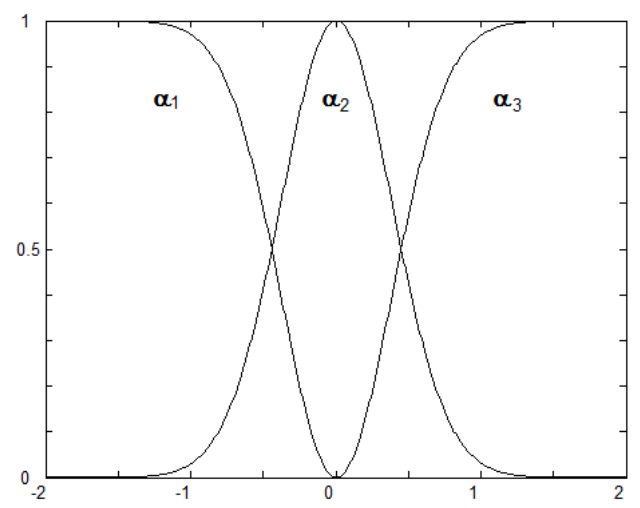

Şekil 5. Biçin bulanık alt kümeler

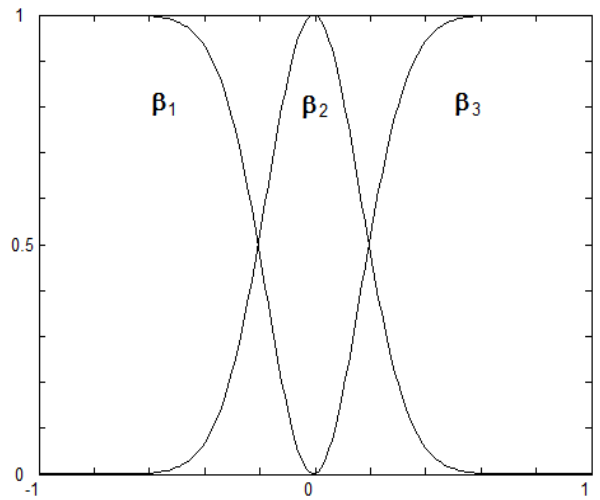

ABSS için test aşamasına ait sonuçlar ise Koç ve ark., (2019) tarafindan aynı eğitme ve test kümelerine dayalı olarak geliştirilen Mamdani ve Sugeno tipi bulanık sistemlerle birlikte klasik etkileşim parametreleri $(\alpha, \beta$ ve $\Delta \eta)$ ve refraktif indeks yöntemleriyle karşılaştırmalı olarak Tablo 2'de verilmiştir.
Çalışmanın ortaya koyduğu bulgular aşağıda kısaca özetlenmiştir: Klasik etkileşim parametreleri $\alpha, \beta$ ve $\Delta \eta$ değerlerine göre doğru sinıflandırma oranları sirasıyla $\% 77.5,82.5$ ve 65 olarak elde edilmesine karşıı bu yöntemlerin birbiriyle uyumlu olmayan kararlara neden olduğu görülmektedir. Bu durum, daha önce detaylı olarak tartışıldığı (Koç ve ark.,2019) için burada yeniden ele alınmayacak olup elde edilen sonuçlar "bulanıklık altında karışabilirlik analizinin" alternatif yollarla iyileştirilebileceğini (Koç ve ark.,2019) teyit eder niteliktedir: Refraktif indeks yöntemiyle alınan kararlar (KRZ veya KR) dikkate alındığında ABSS tipi modelin Sugeno ve Mamdani tipi sistemlere kiyasla daha başarılı olduğu görülmektedir; Mamdani ve Sugeno tipi sistemler için doğru sınıflandırma oranı sırasıyla $\% 85$ ve $\% 73$ (Koç ve ark.,2019) iken ABSS için bu değer \% 90 olarak elde edilmiştir (Tablo 2). Burada, klasik bulanık sistemler (Sugeno ve Mamdani tipi) ile ABSS tipi modellerin, bulanık kural tabanlı modellemenin iki farklı yaklaşımını temsil ettiği söylenebilir. Sugeno ve Mamdani tipi sistemler uzman bilgisine dayalı ve sabit kural tabanlı modeller iken ABSS tipi modeller kural çıkarımını, bir veri tabanı üzerinden, kendiliğinden yapabilen; dolayısıyla göz önüne alınan bir sistemin davranışı modelleyebilen araçlar olarak değerlendirilebilir. Bu özellikleri nedeniyle, bu çalışmada da gösterildiği üzere klasik bulanık sistemlere (Sugeno ve Mamdani tipi) kıyasla, ABSS tipi bir model daha az sayıda bulanık kurala ihtiyaç duyması nedeniyle daha anlaşılabilir ve daha esnek bir modelleme aracı olarak kabul edilebilir ve bu durum, önerilen bulanık sinıflandırma modelinin daha az sayıda bulanık kural (dört adet) yardımıyla daha yüksek tahmin performansına ulaşmasının da nedeni olarak görünmektedir. $\mathrm{Bu}$ çalışma, ABSS tipi bir bulanık sistemin hibrit bir yaklaşım çerçevesinde guguk kuşu aramasına dayalı olarak bir optimizasyon problemi şeklinde formüle edilebileceğini ve guguk kuşu aramasının bu kapsamda uygun bir arama algoritması olduğunu da göstermektedir. 
Tablo 2. Bulanık sistemler, etkileşim parametreleri ve refraktif indeks yöntemleriyle elde edilen karışabilirlik/karışmazlık karar/sinuflandırma durumlar

\begin{tabular}{|c|c|c|c|c|c|c|c|}
\hline $\begin{array}{l}\text { Sira } \\
\text { No }\end{array}$ & $\alpha^{*}$ & $\beta^{*}$ & $\Delta \eta *$ & $\begin{array}{c}\text { Mamdani } \\
\text { tipi } \\
\text { sistem* }\end{array}$ & $\begin{array}{l}\text { Sugeno } \\
\text { tipi } \\
\text { sistem* }\end{array}$ & ABSS & $\begin{array}{l}\text { Refraktif } \\
\text { indeks* }\end{array}$ \\
\hline 77 & KR & KR & KRZ & KRZ & KR & KR & KR \\
\hline 80 & KR & KR & KRZ & KRZ & KR & KR & KR \\
\hline 74 & KRZ & KRZ & KRZ & KRZ & KRZ & KRZ & KRZ \\
\hline 15 & KRZ & KRZ & KRZ & KRZ & KRZ & KRZ & KRZ \\
\hline 31 & KRZ & KRZ & KRZ & KRZ & KRZ & KRZ & KRZ \\
\hline 93 & KR & KR & KRZ & KRZ & KRZ & KRZ & KRZ \\
\hline 76 & KR & KR & KRZ & KR & KR & KR & KR \\
\hline 78 & KR & KR & KRZ & KRZ & KR & KR & KR \\
\hline 60 & KR & KR & KRZ & KRZ & KRZ & KR & KR \\
\hline 28 & KR & KR & KRZ & KRZ & KR & KR & KRZ \\
\hline 99 & KR & KR & KRZ & KRZ & KRZ & KRZ & KRZ \\
\hline 17 & KRZ & KRZ & KRZ & KRZ & KRZ & KRZ & KRZ \\
\hline 9 & KR & KR & KRZ & KRZ & KR & KRZ & KR \\
\hline 51 & KR & KR & KRZ & KR & KRZ & KR & KR \\
\hline 7 & KR & KR & KRZ & KRZ & KR & KR & KR \\
\hline 29 & KR & KR & KRZ & KRZ & KR & KR & KRZ \\
\hline 13 & KR & KRZ & KRZ & KRZ & KRZ & KRZ & KRZ \\
\hline 47 & KRZ & KRZ & KRZ & KRZ & KRZ & KRZ & KRZ \\
\hline 66 & KR & KR & KRZ & KRZ & KR & KR & KR \\
\hline 37 & KRZ & KRZ & KRZ & KRZ & KRZ & KRZ & KRZ \\
\hline 25 & KRZ & KRZ & KRZ & KRZ & KRZ & KRZ & KRZ \\
\hline 5 & KR & KR & KRZ & KRZ & KR & KR & KR \\
\hline 38 & KRZ & KRZ & KRZ & KRZ & KRZ & KRZ & KRZ \\
\hline 59 & KR & KR & KRZ & KRZ & KR & KR & KR \\
\hline 91 & KR & KR & KRZ & KRZ & KRZ & KRZ & KRZ \\
\hline 86 & KRZ & KRZ & KRZ & KRZ & KRZ & KRZ & KRZ \\
\hline 94 & KR & KR & KRZ & KRZ & KRZ & KRZ & KRZ \\
\hline 44 & KRZ & KRZ & KRZ & KRZ & KRZ & KRZ & KRZ \\
\hline 21 & KRZ & KRZ & KRZ & KRZ & KRZ & KRZ & KRZ \\
\hline 72 & KRZ & KRZ & KRZ & KRZ & KRZ & KRZ & KRZ \\
\hline 40 & KRZ & KRZ & KRZ & KRZ & KRZ & KRZ & KRZ \\
\hline 32 & KRZ & KRZ & KRZ & KRZ & KRZ & KRZ & KRZ \\
\hline 62 & KR & KR & KRZ & KRZ & KRZ & KR & KR \\
\hline 12 & KRZ & KRZ & KRZ & KRZ & KRZ & KRZ & KRZ \\
\hline 19 & KRZ & KRZ & KRZ & KRZ & KRZ & KRZ & KRZ \\
\hline 57 & KR & KR & KRZ & KRZ & KR & KR & KR \\
\hline 1 & KR & KR & KRZ & KR & KR & KR & KR \\
\hline 34 & KRZ & KRZ & KRZ & KRZ & KRZ & KRZ & KRZ \\
\hline 79 & KR & KR & KRZ & KRZ & KR & $\mathrm{KR}$ & KRZ \\
\hline 100 & KR & KRZ & KRZ & KRZ & KRZ & KRZ & KRZ \\
\hline
\end{tabular}

\section{Sonuç}

Bu çalışmada, önerilen ABSS tipi model polimer-polimer karışımların karışabilirlik/karışmazlık tahminine SA/PEG karışımlar örneğinde uygulamıştır. Elde edilen sonuçlar, önerilen modelin klasik (bulanık) sistemlere kıyasla daha yüksek tahmin performansına sahip olduğunu; polimerpolimer karışabilirlik tahminlerinin viskometrik verilerin bulanıklık altında işlenmesi yoluyla iyileştirilebileceğini ve bunun karar almayı kolaylaştırabileceğini göstermiştir.

\section{Kaynakça}

Ding, X., Xu, Z., Cheung, N.J., Liu, X., Parameter estimation of Takagi-Sugeno fuzzy system using heterogeneous cuckoo search algoritm, Neurocomputing 151, 1332-1342, 2015.

Ebenezer, N.G.R., Ramabalan, S. Navaneethasanthakumar, S., Advanced design optimization on straight bevel gears pair based on nature inspired algorithms, SN Applied Sciences $1: 1155,2019$.

Hodashinsky, I.A., Minina, D. Yu., and Sarin, K.S., Identification of the parameters of fuzzy 
approximators and classifiers based on the cuckoo search, Optoelectronics, Instrumentation and Data Processing, 51:3, 234-240, 2015.

Koç, D.İ, Koç, M.L., Fuzzy viscometric analysis of polymer-polymer miscibility based on fuzzy regression, Chemometrics and Intelligent Laboratory Systems 157, 58-66, 2016.

Koç, D.İ, Özel Ergün, N., Koç, M.L. Prediction of miscibility of sodium alginate/poly (ethyleneglycol) blends by fuzzy systems, Journal of the Faculty of Engineering and Architecture of Gazi University 34:2 635-646, 2019.

Kumar, M., Rawat, T.K., Optimal fractional delay-IIR filter design using cuckoo search algorithm. ISA Transactions 59:39-54, 2015.

Mamdani, E.H., Application of fuzzy logic to approximate reasoning using linguistic systems, Fuzzy Sets and Systems 26, 1182-1191, 1977.

Nakashima, T.,Schaefen, G., Yokota, Y., Ishibuchi, H., A weighted fuzzy classifier and its application to image processing tasks, Fuzzy Sets and Systems 158, 284294, 2007.

Nawi, N.M., Khan, A., Rehman, M.Z., A new Levenberg Marquardt based backpropagation algorithm trained with cuckoo search, Procedia Technology 11, 18-23, 2013.

Ozkaya, U., Seyfi, L. A novel fuzzy logic model for intelligent traffic systems. Electronics World, 122(1960), 36-39, 2016.

Samir, K., Brahim, B., Capozucca, R., Wahab, M.A., Damage detection in CFRP composite beams based on vibration analysis using proper orthogonal decomposition method with radial basis functions and zuckoo search algorithm, Composite Structures, 187, 344-353, 2018.

Takagi, T., Sugeno, M., Fuzzy identification of systems and its applications to modeling and control, IEEE Transactions on Systems, Man and Cybernetics 15(1), 116-132, 1985.

Yang, X.S., Deb, S., Multi objective cuckoo search for design optimization, Computers $\square$ Operations Research 40, 1616-1624, 2013.

Yang, X.S., Deb, S., Cuckoo search: Recent advances and applications, Neural Computing and Applications 24, 169-174, 2014. 